\title{
Uso del procesamiento digital de imágenes para la extracción de datos de medidas experimentales publicados en formato gráfico
}

\section{Use of digital image processing for the extraction of experimental measurement data published in graphic format}

PEZA-ORTIZ, Edebaldo $\dagger^{*}$, TORRES-VALLE, Jose Bernardo, GARCÍA-TRINIDAD, Enrique y GONZÁLEZ-RAMOS, Alma Delia

Universidad Tecnológica Fidel Velázquez, División de Mantenimiento Industrial y Mecatrónica

ID 1 ${ }^{\mathrm{er}}$ Autor: Edebaldo, Peza Ortiz, / ORC ID: 0000-0003-0236-883X, CVU CONACYT ID: 778771

ID $1^{\mathrm{er}}$ Coautor José Bernardo, Torres Valle / ORC ID: 0000-0002-4302-1640, Researcher ID Thomson: W-7170-2019

ID $2^{\mathrm{do}}$ Coautor: Enrique, García Trinidad / ORC ID: 0000-0003-2875-0500

ID $3^{\text {er} C o a u t o r: ~ A l m a ~ D e l i a, ~ G o n z a ́ l e z ~ R a m o s ~ G o r a ~ / ~ O R C ~ I D: ~ 0000-0001-5150-5058, ~ R e s e a r c h e r ~ I D ~ T h o m s o n: ~ U-9209-~}$ 2018, CVU CONACYT ID: 950682

DOI: $10.35429 /$ JOCT.2019.10.3.12.21

Recibido 16 de Marzo, 2019; Aceptado 30 Junio, 2019

\begin{abstract}
Resumen
En este artículo se propone un método como alternativa para obtener datos de medidas experimentales, ante la ausencia de equipo de laboratorio para realizar pruebas, en un formato adecuado para efectuar operaciones de índole matemático con el fin de utilizarlos como información para validar: hipótesis, modelos constitutivos y/o teorías de investigaciones enfocadas al desarrollo tecnológico. El método propuesto utiliza como principal herramienta la técnica de segmentación de imágenes por crecimiento de regiones por agrupamiento de pixeles y la normalización de las coordenadas de los las posiciones de los pixeles extraídos a la escala de los ejes en la figura correspondiente. La segmentación de la imagen separa las coordenadas de los pixeles que forman los ejes y de las curvas, las coordenadas de los pixeles de las curvas se normalizan a la escala de los ejes. El método se prueba con imágenes del resultado de pruebas experimentales del comportamiento esfuerzo-deformación recuperadas de [1]. Los resultados de la extracción de datos se grafican y se obtiene los promedios de cada curva extraída, así como la desviación estándar. Se verifica que los datos obtenidos pueden ser de utiliza dos para corroborar o respaldar hipótesis en un amplio margen de investigaciones.
\end{abstract}

Segmentación, Extracción, Normalización

\begin{abstract}
In this article, we propose a method as an alternative to obtain experimental measurement data, in the absence of laboratory equipment to perform tests, in a suitable format to perform mathematical operations in order to use them as information to validate: hypotheses, models constitutive and / or research theories focused on technological development. The proposed method uses as a main tool the image segmentation technique by region growth by pixel grouping and the normalization of the coordinates of the positions of the pixels extracted to the axis scale in the corresponding figure. The segmentation of the image separates the coordinates of the pixels that form the axes and the curves, the coordinates of the pixels of the curves are normalized to the scale of the axes. The method is tested with images of the result of experimental tests of stress-strain behavior recovered from [1]. The results of the data extraction are plotted and the averages of each curve extracted as well as the standard deviation are obtained. It is verified that the data obtained can be used to corroborate or support hypotheses in a wide range of investigations.
\end{abstract}

Segmentation, Extraction, Normalization

Citación: PEZA-ORTIZ, Edebaldo, TORRES-VALLE, Jose Bernardo, GARCÍA-TRINIDAD, Enrique y GONZÁLEZRAMOS, Alma Delia. Uso del procesamiento digital de imágenes para la extracción de datos de medidas experimentales publicados en formato gráfico. Revista de Tecnologías Computacionales. 2019. 3-10: 12-22

$\dagger$ Investigador contribuyendo como primer autor. 


\section{Introducción}

Una de las actividades fundamentales en el ámbito de la investigación científica y desarrollo tecnológico es la toma de medidas en pruebas experimentales. Una limitante a la que instituciones de educación superior se enfrentan para realizar desarrollo tecnológico es la carencia o falta de instrumentos de medida para adquirir datos de eventos experimentales. En las publicaciones científicas es común encontrar los resultados de las investigaciones en formato gráfico. En el procesamiento de imágenes, como rama de la ciencia de la computación, se han desarrollado técnicas de segmentación de imágenes que permiten, a partir de una imagen, diferenciar los objetos presentes en una escena, tomando en consideración estos dos aspectos se plantea la posibilidad de extraer, a partir de gráficos publicados en artículos de investigación, datos medidos en pruebas experimentales, de esta forma contar información experimental para comparar con información generada de manera analítica y con ello validar las hipótesis propuestas.

En este trabajo se propone el uso de la técnica de segmentación de imágenes por crecimiento supervisado de regiones para extraer datos de medidas experimentales a partir de imágenes obtenidas de publicaciones científicas. Como ejemplo del rendimiento del método propuesto para extraer datos experimentales se utilizan los gráficos de las medidas experimentales publicados en [1], los cuales son gráficos que muestran las medidas del comportamiento esfuerzo-deformación de una barra de PUSMP-MM2520, poliuretano que posee la capacidad programación y recuperación de forma. En los siguientes apartados se expone la metodología utilizada para segmentar las imágenes y los resultados de someter estas imágenes al procesamiento computacional para separar las gráficas que se muestran dentro de una figura.

\section{Segmentación de imágenes por regiones}

En el campo del procesamiento de imágenes los temas de mayor interés son identificar patrones u objetos en imágenes "complejas", defino de forma muy simple y particular como imagen compleja a toda aquella cuyas características dificultan extraer la información buscada en la imagen.
Dentro de estas características invariablemente se encuentran la resolución de la imagen, el tamaño, el tipo de imagen, es decir binaria, en escala de grises, RGB, etc. y de forma muy particular las características de los objetos que están dentro de la imagen.

La segmentación de imágenes es el proceso de dividir una imagen en varios grupos de píxeles. Esto se realiza asignado una etiqueta a cada píxel de la imagen de forma que los píxeles que compartan la misma etiqueta también tendrán ciertas características visuales similares. Cada uno de los píxeles de una región poseen las mismas características o similares, como el color, la intensidad o la textura. La segmentación se usa para localizar objetos, dentro de una imagen.

La segmentación de imágenes en regiones se basa en el agrupamiento de píxeles en zonas con características similares. Para una región dada, en general, los píxeles pertenecen a un objeto simple. A este conjunto de puntos conectados que pertenecen al mismo objeto se llama región. La segmentación de imágenes por regiones se puede definir como [2]:

Sea $\mathrm{R}$ la representación en una región completa de una imagen. Se contempla la segmentación como un proceso que divide a $\mathrm{R}$ en $n$ subregiones, $\mathrm{R} 1, \mathrm{R} 2, \ldots, \mathrm{Rn}$ tal que

$$
\begin{aligned}
& \bigcup_{i=1}^{n} R_{i}=R \\
& R_{i} \text { es una regiónconexa, } \mathrm{i}=1,2, \ldots, \mathrm{n} \\
& R_{i} \cap R_{j}=\emptyset \text { para todo } i y j, i \neq j \\
& P\left(R_{i}\right)=\text { Verdaderoparai }=1,2, \ldots, n \\
& P\left(R_{i} \cup R_{j}\right)=\text { Falsoparai } \neq j
\end{aligned}
$$

Donde $P\left(R_{i}\right)$ es una propiedad de los puntos del conjunto $R_{i}$ y $\emptyset$ es el conjunto vacío. La ecuación (1) expresa que la segmentación debe ser completa, es decir, cada pixel debe pertenecer a una región, la expresión (2) indica que los puntos de región deben ser conexos, la expresión (3) indica que las regiones son disjuntas, la expresión (4) indica que todos los pixeles que forman una región cumplen una misma propiedad, y la expresión (5) expresa que todas las regiones son distintas entre sí [3]. 


\section{Algoritmo para extraer los datos}

Se han desarrollado una amplia variedad de técnicas y metodologías para separar grupos de información dentro de una imagen, las gráficas de las cuales se buscan obtener los valores de esfuerzo-deformación no representan gran complejidad debido a que las curvan están perfectamente diferenciadas por intensidad de color, por posición y por continuidad. Por lo que no ha sido necesario implementar técnicas avanzadas para extraer los datos que en ellas se muestran.

Las etapas necesarias para extraer los datos de interés se identifican como: 1) Digitalizar la imagen en el formato más conveniente según el lenguaje y librerías de programación que se utilice, en este trabajo el formato que se le da a las imágenes donde se ilustran los gráficos experimentales es JGP. 2) Editar la imagen, esta etapa consiste en eliminar de la imagen aspectos no deseados de la imagen tal como líneas, símbolos y ajustar el tamaño de la imagen para que la figura del gráfico sea lo único presente. Se recomienda realizar esta etapa con una herramienta de diseño gráfico siempre teniendo la debida precaución de no modificar la relación de pixeles entre los ejes del gráfico y la o las curvas mostradas en la figura, y 3) Aplicar el procedimiento de segmentación a la figura editada.

El paso dos es fundamental para el procedimiento, el principal dato en el que se apoya este, para extraer los datos de las figuras de gráficos, son: las coordenadas de cada pixel en la figura, los colores de los pixeles (curvas y ejes), el número de pixeles y la vecindad de pixeles. La escala de los ejes es de suma importancia para reconstruir las curvas, así también para realizar operaciones sobre las mismas tal como interpolaciones, extrapolaciones, cálculo de medias, etc. Los pasos seguidos para la segmentación en este programa son los siguientes:

1. La imagen de color de entrada se representará de manera aproximada utilizando 25 contenedores.

2. La representación aproximada utiliza la información espacial de un proceso de ventanas basado en histograma.

3. K-Means se usa para agrupar los datos de las curvas en diferentes categorías o regiones.

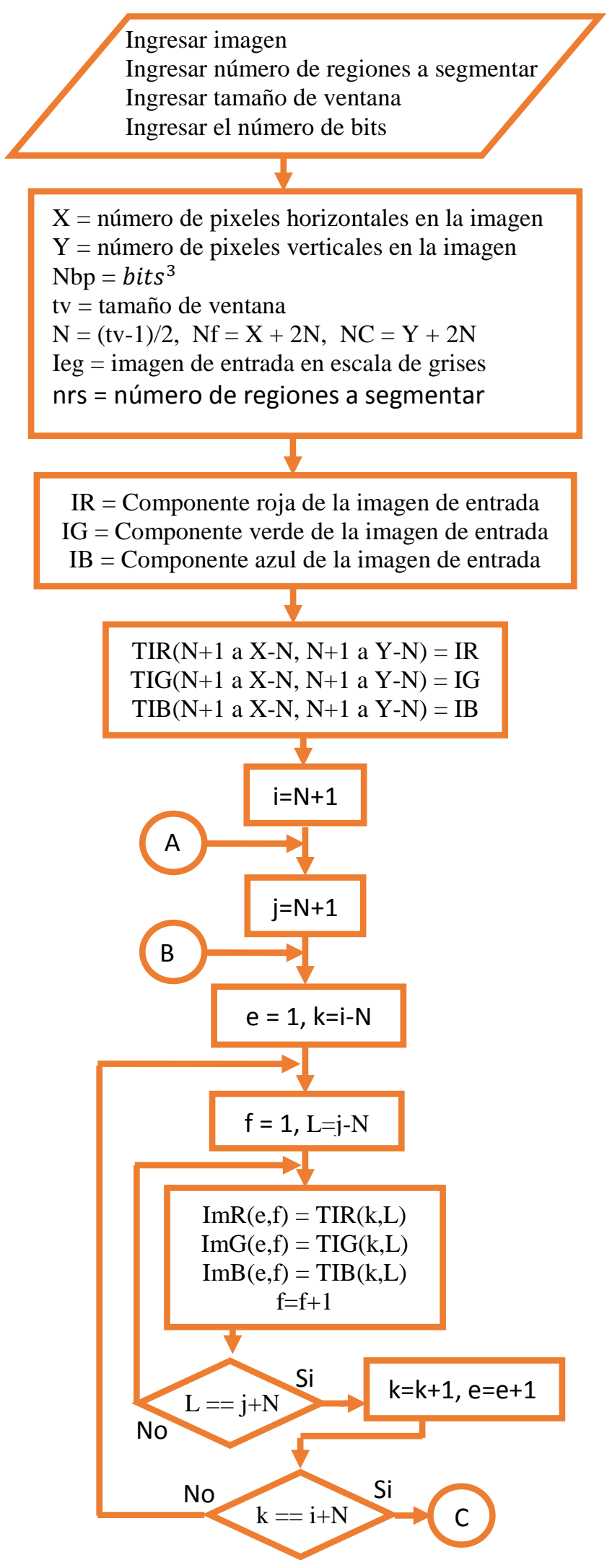

Figura 1.1 Algoritmo para extraer los datos mostrados en las gráficas de las medidas experimentales. Parte 1 


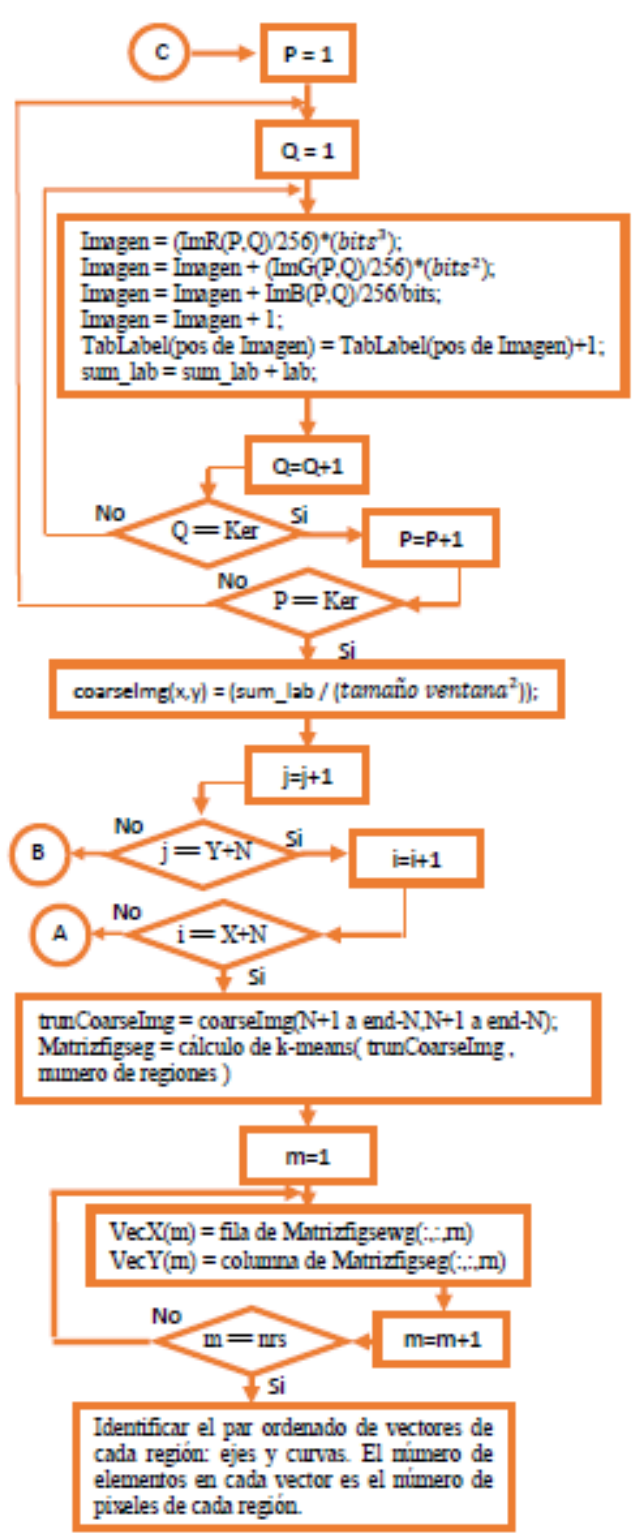

Figura 1.2 Algoritmo para extraer los datos mostrados en las gráficas de las medidas experimentales. Parte 2

Las figuras 1.1 y 1.2 ilustran el algoritmo seguido para segmentar las regiones en la gráfica y extraer los datos de las figuras. Para separar las regiones se utiliza el procedimiento de $\mathrm{K}$-medias, pero se puede utilizar cualquier método para agrupar datos. Con el fin de reducir el costo computacional se transforma la imagen de color a escala de grises, donde según el color del pixel se asigna un nivel de intensidad de gris, posteriormente se calcula el histograma de la imagen en escala de grises para identificar los niveles de gris de cada región.

Como resultado de aplicar el algoritmo ilustrado por el diagrama de flujo de las figuras 1.1 y 1.2 se obtienen vectores con las coordenadas de los pixeles de cada región en formato de número entero.
Con estos valores se está en condiciones de trasformar el valor de coordenada del pixel por el valor de la magnitud del esfuerzo o deformación al normaliza los vectores de coordenadas a las escalas de los ejes respectivamente. En las siguientes secciones se describen las figuras utilizadas para probar este algoritmo y los resultados correspondientes.

\section{Imágenes de prueba}

Como imágenes de prueba se toman las figuras de medidas experimentales publicadas en [1].La figura 1 es el resultado que se obtiene al medir el valor del esfuerzo versus la deformación que experimenta una barra de PUSMP-MM2520, esta figura muestra que en este material la curva fuerza-deformación posee un comportamiento de histéresis, durante la carga los valores de esfuerzo y deformación parten de cero y estos aumentan de manera aparentemente proporcional, cuando la deformación alcanza el valor de 0.7 la barra de polímero se libera de la carga, entonces los esfuerzos dentro de la barra se liberan hasta alcanzar casi el valor de cero cuando la deformación disminuye de 0.7 a 0.15 aproximadamente.

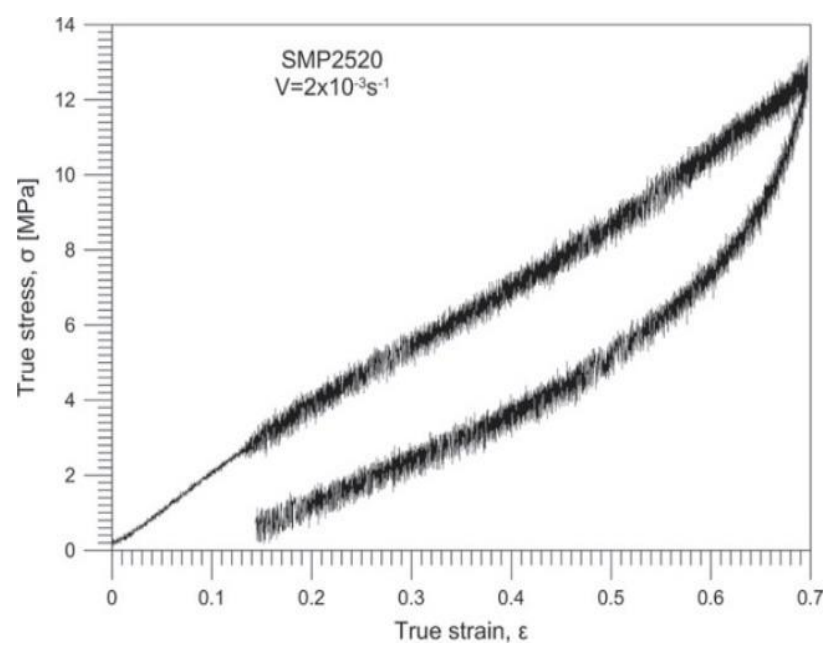

Figura 1 Curva esfuerzo vs deformación obtenida para una velocidad de deformación $2 \times 10-3$ s-1. Recuperado de [1]

Es relevante observar que la gráfica es afectada por ruido, el ruido influye en los resultados que se obtienen al recuperar las coordenadas de los pixeles pues le otorga un grosor mayor al de un píxel a la curva, esto ocasiona que en la recuperación de los datos se obtenga una nube de puntos para pequeños segmentos de la curva, esta nube de puntos se presenta en cada curva recuperada con el procedimiento utilizado en este trabajo. 
La figura dos muestra cuatro curvas que indican diferentes magnitudes de esfuerzo y deformación, la figura incluye, además de las curvas, los ejes, las etiquetas de los ejes, la escala de los ejes y texto que indica la velocidad a la que se deformo la barra de polímero.

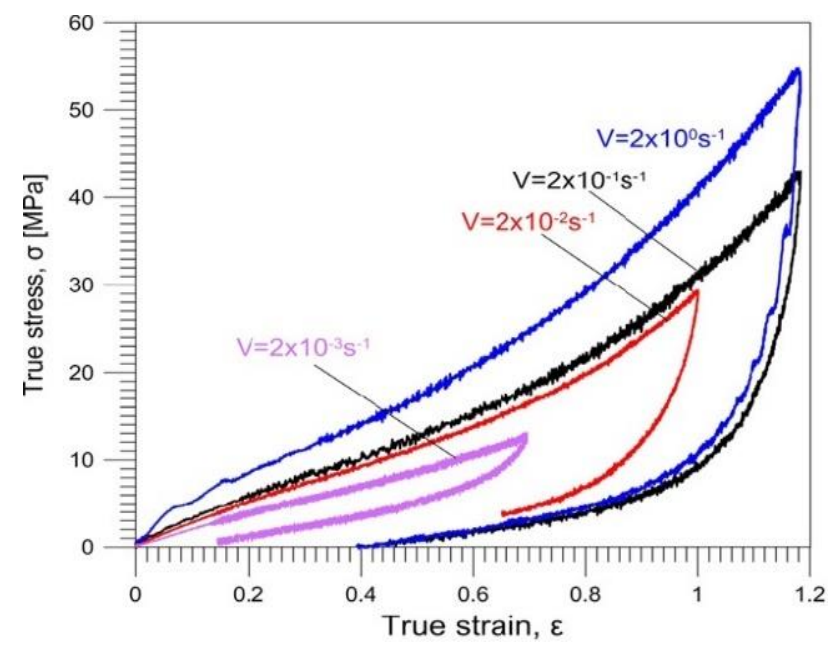

Figura 2 Curvas Esfuerzo vs deformación obtenidas para diversas velocidades y rangos de deformación en condiciones ambientales. Recuperado de [1]

En la figura 2, además de número de curvas, la escala de los ejes es distinta a la escala, en relación con el tamaño de la imagen, mostrada en la figura 1 y en ciertas zonas las curvas se traslapan, estas condiciones agregan complejidad al proceso de segmentar las curvas para extraer los datos de cada curva por separado.

En las figuras 3 y 4 se muestran cuatro curvas esfuerzo deformación en cada una, en estas figuras las gráficas se traslapan en mayor o menor grado. En ambas figuras se puede observar texto, es importante eliminar el texto debido a que el color de este coincide con el color de los ejes y/o las curvas.

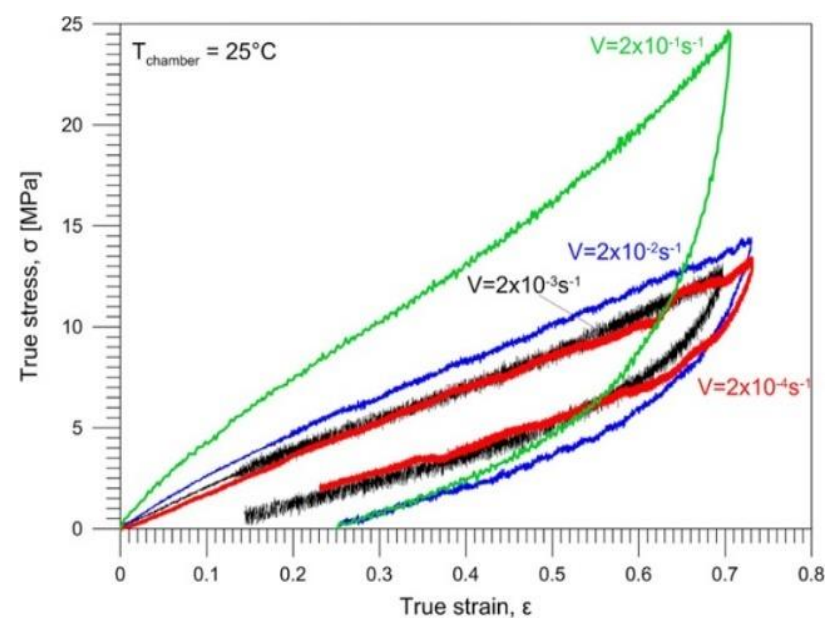

Figura 3 Esfuerzovs deformación, obtenida para diversas velocidades de deformación a temperatura constante de 25 ${ }^{\circ} \mathrm{C}$. Recuperado de [1]

ISSN 2523-6814

ECORFAN® Todos los derechos reservado

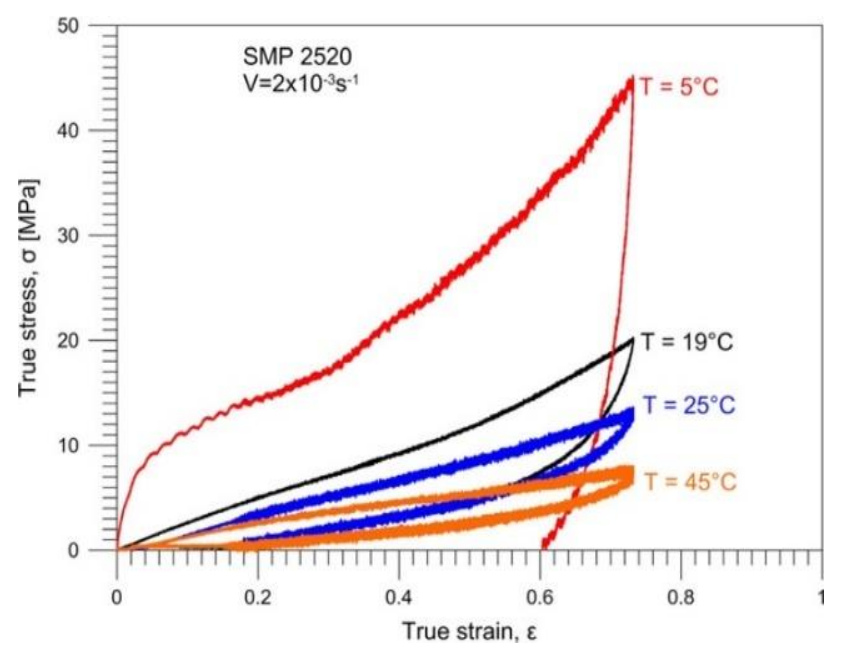

Figura 4 Comparación de curvas de esfuerzo frente a deformación obtenidas a velocidad de deformación constante y diferentes temperaturas. Recuperado de [1]

Este conjunto de imágenes es el que se utiliza para extraer los valores de las curvas esfuerzo-deformación de las pruebas realizadas al material en [1], con tal fin, se procesan las imágenes para separar las curvas, extraer las coordenadas de cada pixel en cada una y obtener un valor representativo del pixel en términos de su posición en la imagen.

\section{Resultados}

Con ayuda del procesamiento de imagen se obtienen matrices con valores de intensidad de los pixeles, donde cada curva tiene un valor en escala de gris y cada nivel de gris corresponde a una región. Una vez separadas las curvas por regiones se extraen las coordenadas de los pixeles, con ayuda de la escala indicada en los ejes se evalúa la posición de cada pixel con respecto a las coordenadas de los pixeles que forman los ejes, con ello se calculan los valores de las curvas.

A continuación, se muestran resultados representativos de los datos obtenidos a partir de las gráficas.

Las figuras $5,7,13$ y 19 son el resultado de aplicar el algoritmo de segmentación de imágenes por crecimiento de regiones a las figuras 1, 2, 3 y 4 respectivamente. Al segmentar las imágenes se separan las curvas y textos en diferentes niveles de gris, los niveles de gris se establecen en función del color de cada elemento en las figuras.

En la figura 5 se visualizan dos regiones, una es el fondo de la figura y la otra región es la que se visualiza en color blanco.

PEZA-ORTIZ, Edebaldo, TORRES-VALLE, Jose Bernardo, GARCÍATRINIDAD, Enrique y GONZÁLEZ-RAMOS, Alma Delia. Uso del procesamiento digital de imágenes para la extracción de datos de medidas experimentales publicados en formato gráfico. Revista de Tecnologías Computacionales. 2019 


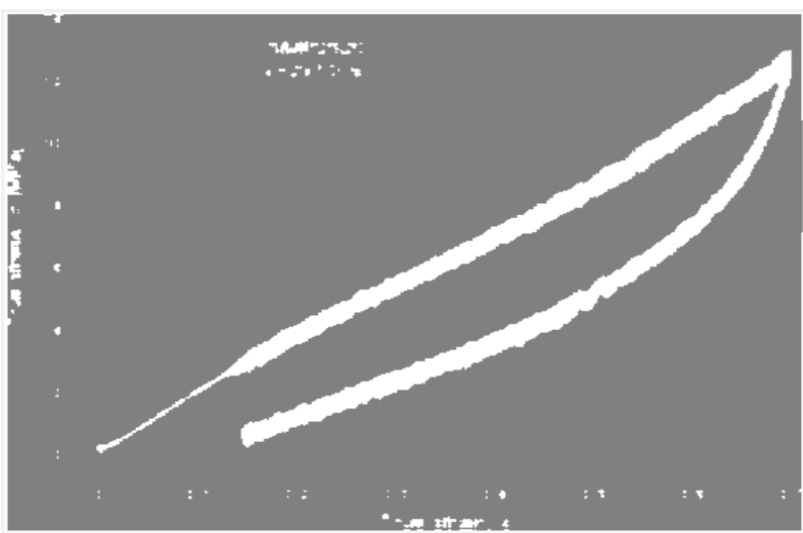

Figura 5 Resultado de segmentar la figura 1 en dos regiones de pixeles

Una vez separadas las figuras en regiones se obtienen las coordenadas de los pixeles como posiciones en una matriz de tantas filas como puntos y dos columnas una para cada coordenada $(x, y)$, posteriormente los valores de posición $(\mathrm{x}, \mathrm{y})$ se normalizan a la escala de los ejes, de esta forma los valores de posición se trasforman en valores con mismas unidades de medida que los ejes, de tal forma que las matrices de posición de los pixeles se convierten en matrices de coordenadas para las magnitudes de esfuerzo y deformación.

Por la naturaleza de las imágenes de [1] donde las curvas ocupan una vecindad de pixeles calculo curvas promedio para cada una y evalúo la desviación típica de los valores extraídos, en cada figura indico la desviación típica. Bajo estas condiciones la desviación se debe al número de pixeles que otorgan grosor a las gráficas, por lo que mayor desviación indica líneas más gruesas que se difuminan con píxeles que reducen su intensidad de color acercándose al valor del color de fondo de las curvas, en este caso el color blanco.

Una vez que se obtienen las matrices de magnitudes se cuenta con el formato adecuado de los datos para realizar operaciones sobre los puntos de las curvas, ya sea obtener promedio o calcular la desviación estándar, por ejemplo.

La figura 6 ilustra el resultado de calcular el promedio de la nube de puntos obtenida de la figura 5 junto con la desviación estándar de la nube de puntos respecto a la media.

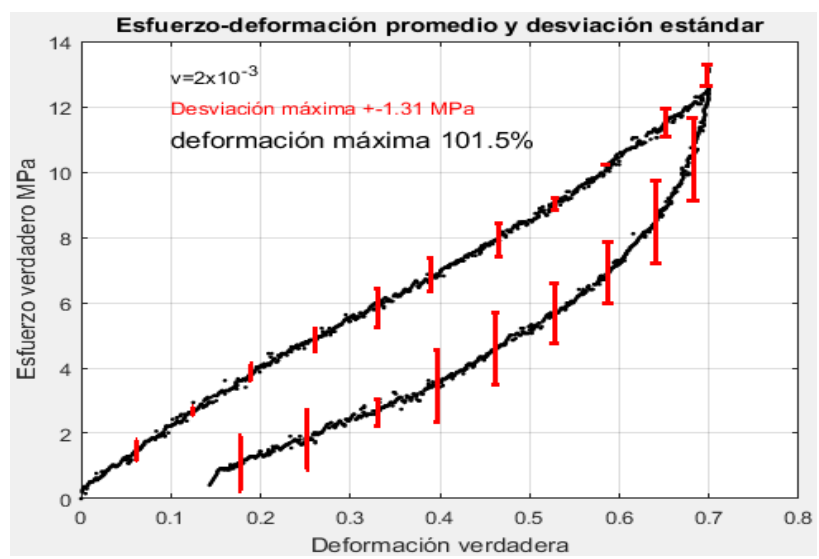

Figura 6 Curva esfuerzo-deformación promedio y barras de error expresado por su desviación estándar de la curva de la figura 5

La figura 7 es la separación de la figura 2 en seis regiones, 1) el fondo, 2) los ejes y sus nombres, 3 a 6 las curvas junto con sus textos.

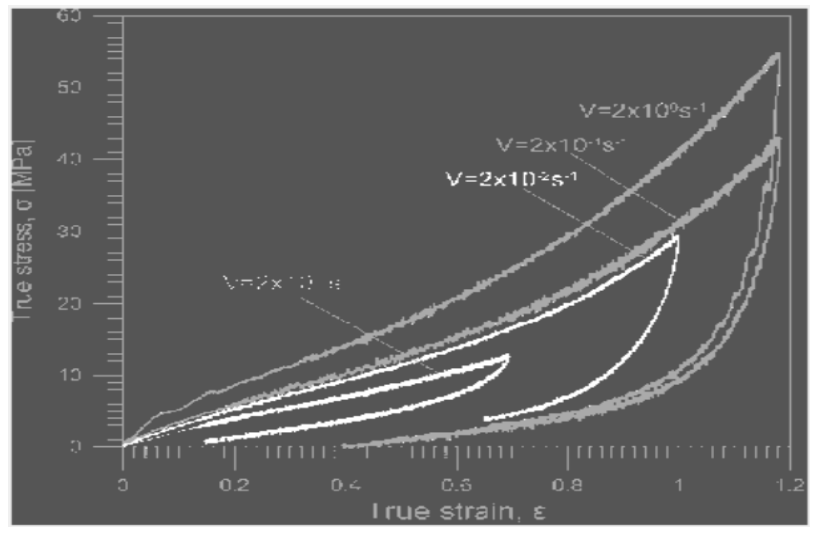

Figura 7 Resultado de segmentar la figura 2 en seis regiones

La figura 8 muestra las nubes de puntos obtenidas, a partir de la figura 7 , parta cada una de las curvas. Aun cuando las gráficas se visualizan en una sola imagen los puntos de cada curva son graficados de manera independiente sobre el mismo plano.

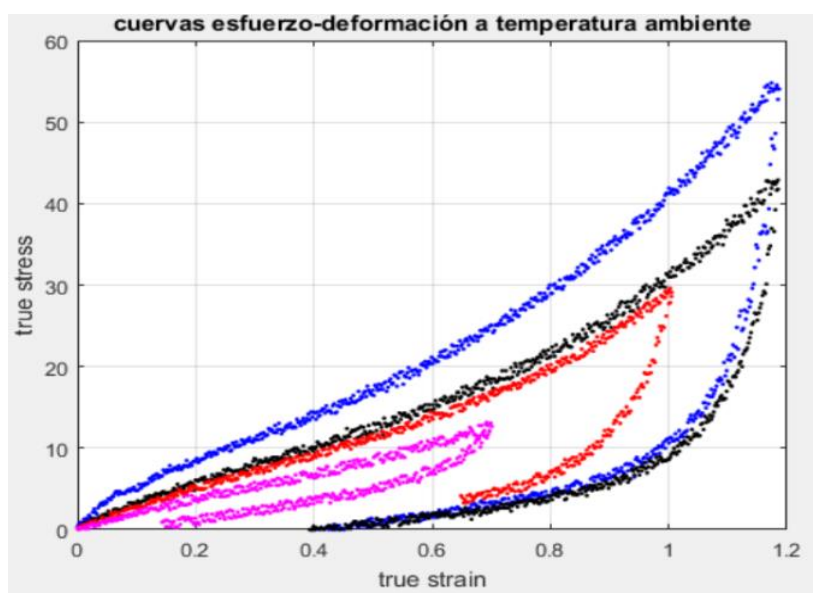

Figura 8 Cruvas esfuerzo vs deformación con los datos extraidos de la figura 7

PEZA-ORTIZ, Edebaldo, TORRES-VALLE, Jose Bernardo, GARCÍATRINIDAD, Enrique y GONZÁLEZ-RAMOS, Alma Delia. Uso del procesamiento digital de imágenes para la extracción de datos de medidas experimentales publicados en formato gráfico. Revista de Tecnologías Computacionales. 2019 
Las figuras 9, 10, 11 y12 muestran las gráficas por separado de las curvas de la figura 8. En cada una de las curvas se ha obtenido el promedio y la desvición estándar. Las lineas verticales en color rojo indican, de forma gráfica, la desviación estándar de la nube de puntos de la gráfica respecto al promedio para el punto sobre el que se encuentra la barra.

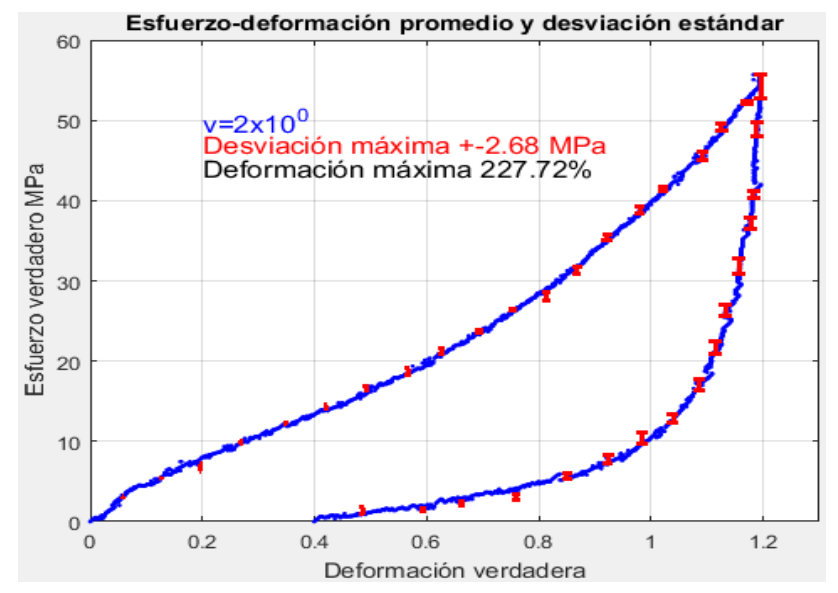

Figura 9 Curva esfuerzo-deformación promedio y barras de error expresado por su desviación estándar de la curva azul en la figura 8. El tiempo para alcanzar la deformación máxima es de 0.6 segundos

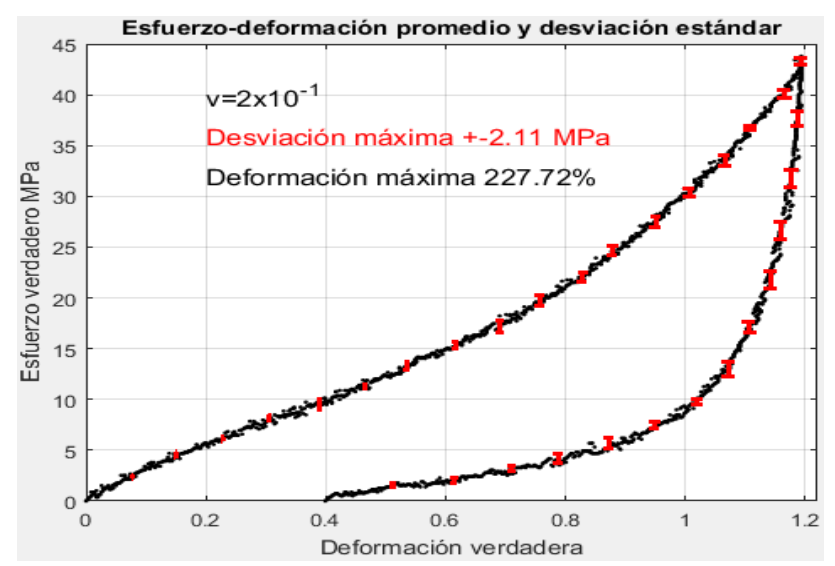

Figura 10 Curva esfuerzo-deformación promedio y barras de error expresado por su desviación estándar obtenida de la figura 8

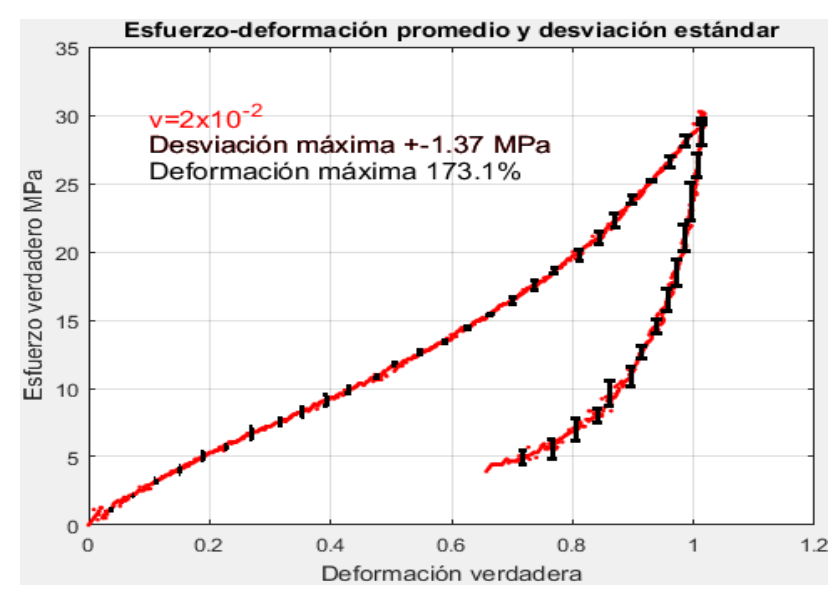

Figura 11 Curva esfuerzo-deformación promedio y barras de error expresado por su desviación estándar de la curva roja en la figura 8

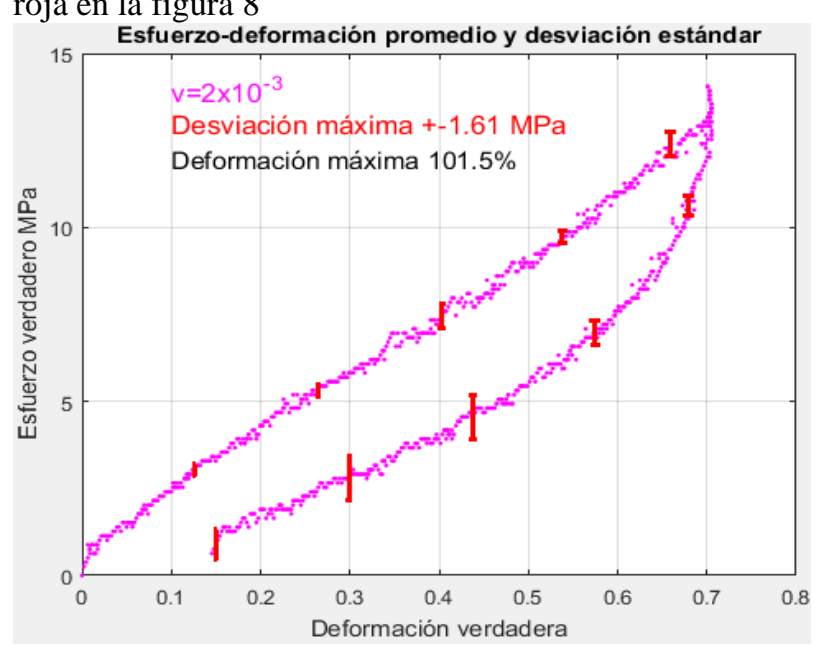

Figura 12 Curva esfuerzo-deformación promedio y barras de error expresado por su desviación estándar de la curva magenta en la figura 8

La figura 13 es la separación de la figura 3 en seis regiones, 1) El fondo, 2) los ejes y sus nombres, 3) a 6) las cruvas y los textos informativos.

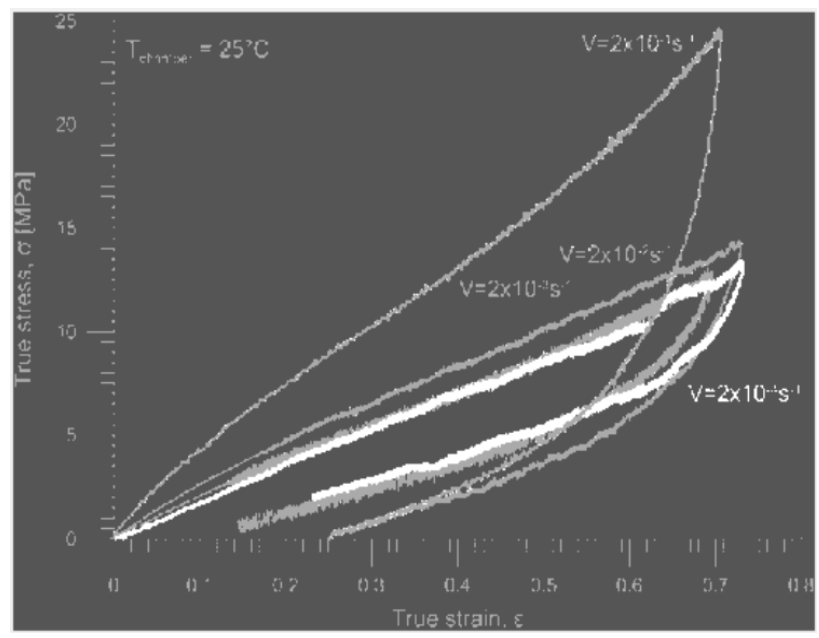

Figura 13 Resultado de segmentar la figura 3 en seis regiones

La figura 14 es el resultado de extraer las coordenadas de los pixeles y de normalizar sus coordenadas con la escala de los ejes.

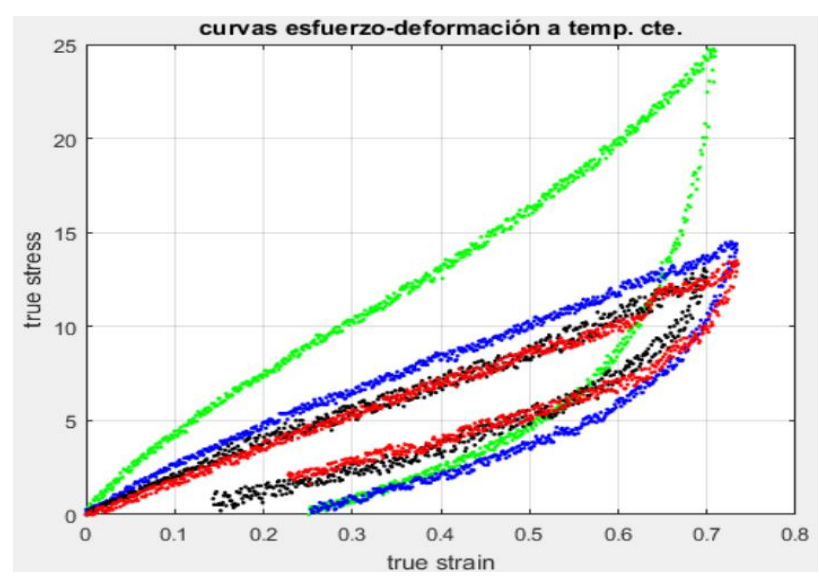

PEZA-ORTIZ, Edebaldo, TORRES-VALLE, Jose Bernardo, GARCÍATRINIDAD, Enrique y GONZÁLEZ-RAMOS, Alma Delia. Uso del procesamiento digital de imágenes para la extracción de datos de medidas experimentales publicados en formato gráfico. Revista de Tecnologías Computacionales. 2019 
Figura 14 Cruvas esfuerzo vs deformación con los datos extraidos de la figura 5

Las figuras 15, 16, 17 y 18 muestran las gráficas por separado de las curvas de la figura 14. En cada una de las curvas se ha obtenido el promedio y la desvición estándar. Las lineas verticales en color rojo indican la desviación estándar de la curva.

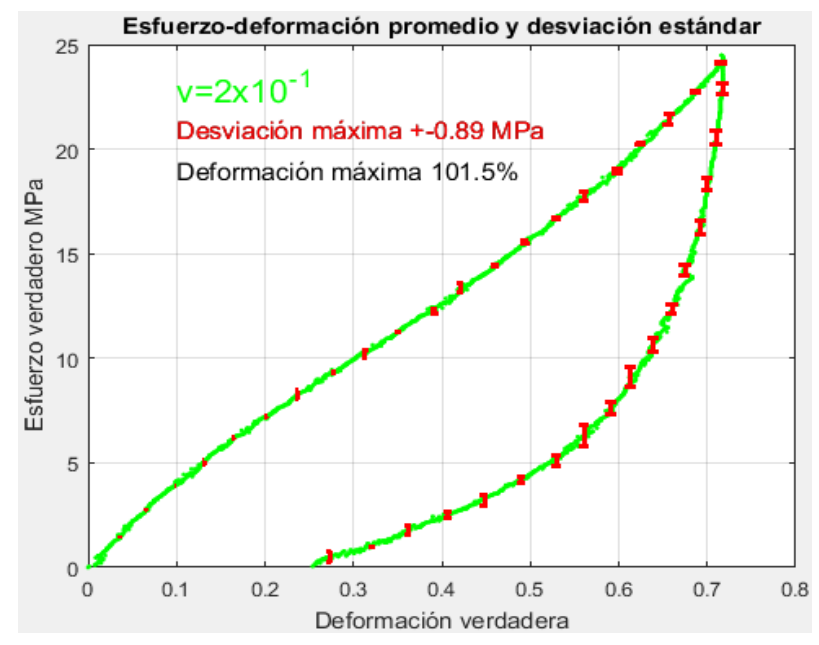

Figura 15 Curva esfuerzo-deformación promedio y barras de error expresado por su desviación estándar

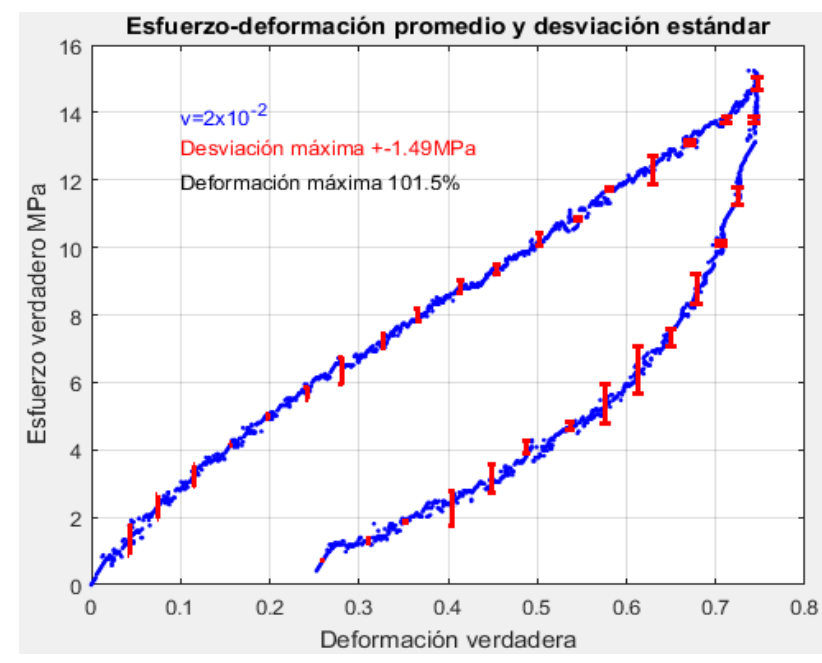

Figura 16 Curva esfuerzo-deformación promedio y barras de error expresado por su desviación estándar

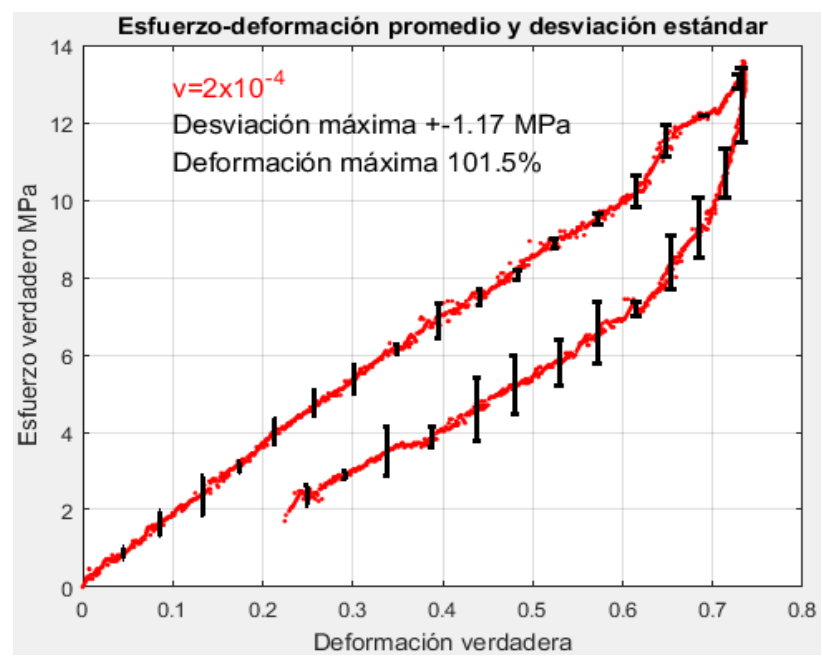

Figura 17 Curva esfuerzo-deformación promedio y barras de error expresado por su desviación estándar

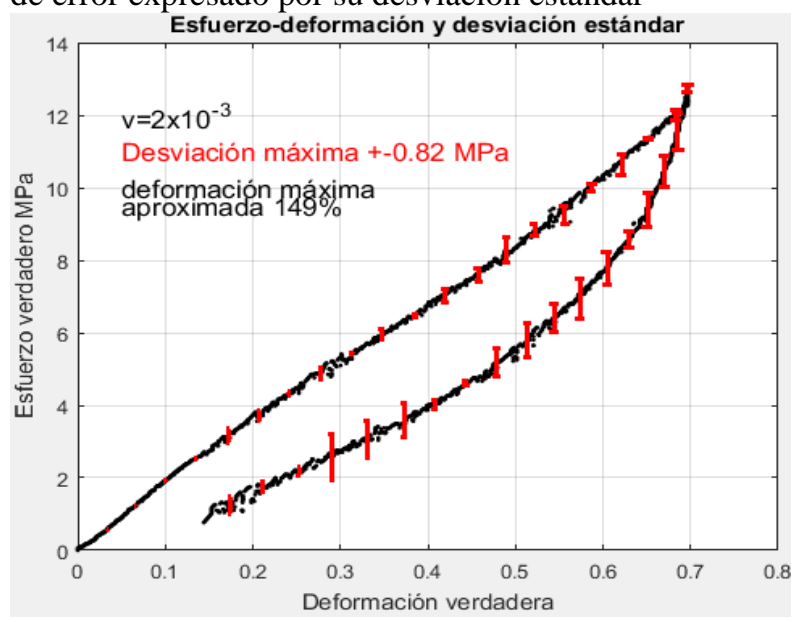

Figura 18 Curva esfuerzo-deformación promedio y barras de error expresado por su desviación estándar

La figura 19 es la separación de la figura 3 en seis regiones, 1) El fondo, 2) los ejes y sus nombres, 3) a 6) las cruvas y los textos informativos.

Es importante notar que los puntos de las cruvas extraidas de las figruas 5, 7, 13 y 19 presentan discontinuidades, sin embargo la cercania entre puntos permite que la interpolación y extrapolación de las curvas sea "suave", excepto en aquellas cruvas donde entre puntos existan "grandes" discontinuidades, tal son los casos en algunas cruvas de las figuras 8 у 20 . 


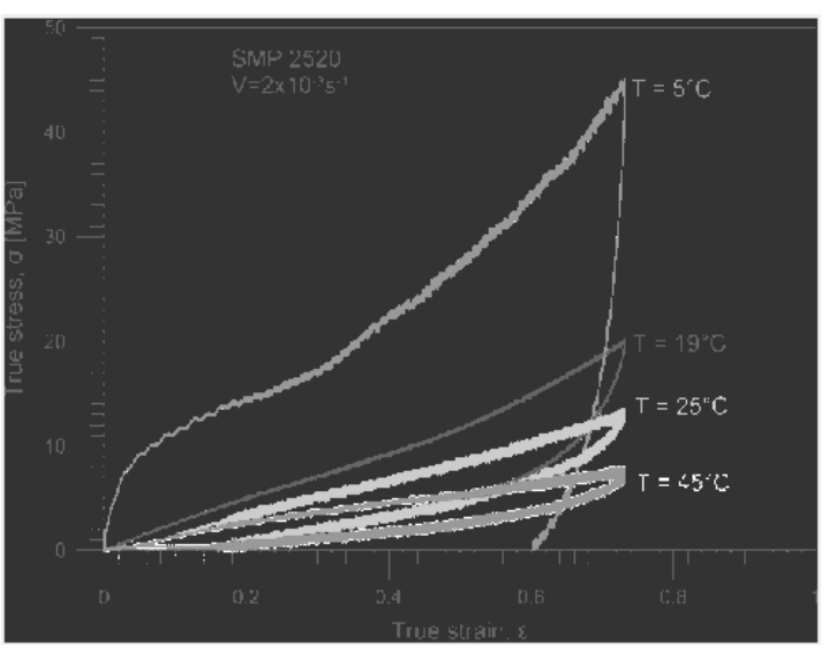

Figura 19 Resultado de segmentar la figura 4 en 6 regiones

La figura 20 muestra las nubes de puntos obtenidas, a partir de la figura 19.

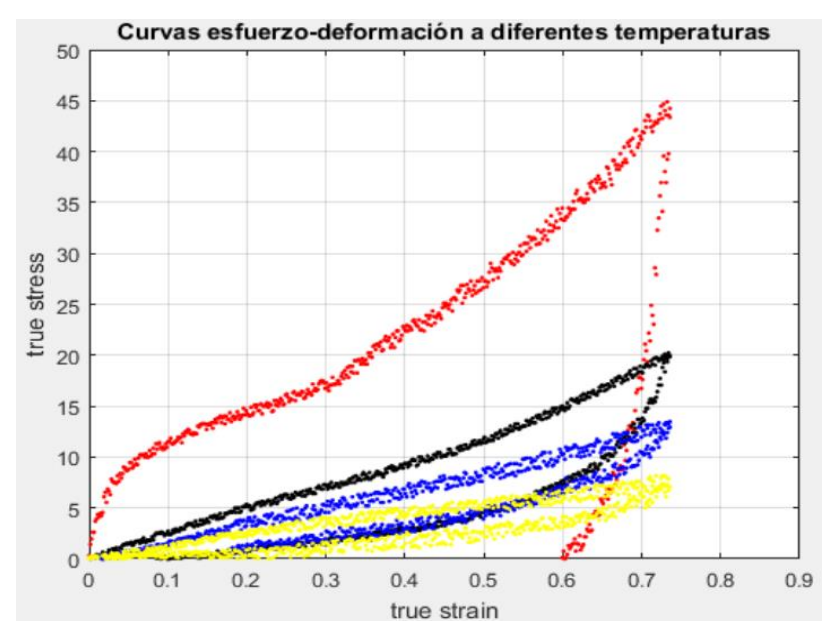

Figura 20 Cruvas esfuerzo deformación con los datos extraidos de la figura 19

En las figuras 21, 22, 23 y 24 se encuentran que en tramos de las cruvas existen discontinuidades a pesar de que son curvas promedio, esto es ocasionado al hecho de que se han interpolado las curvas para "cerrar" los espacios ausentes de puntos, en los resulatdos mostrados en la figrua 20, y que existen secciones de las curvas donde hay una cantidad de puntos muy escasa.

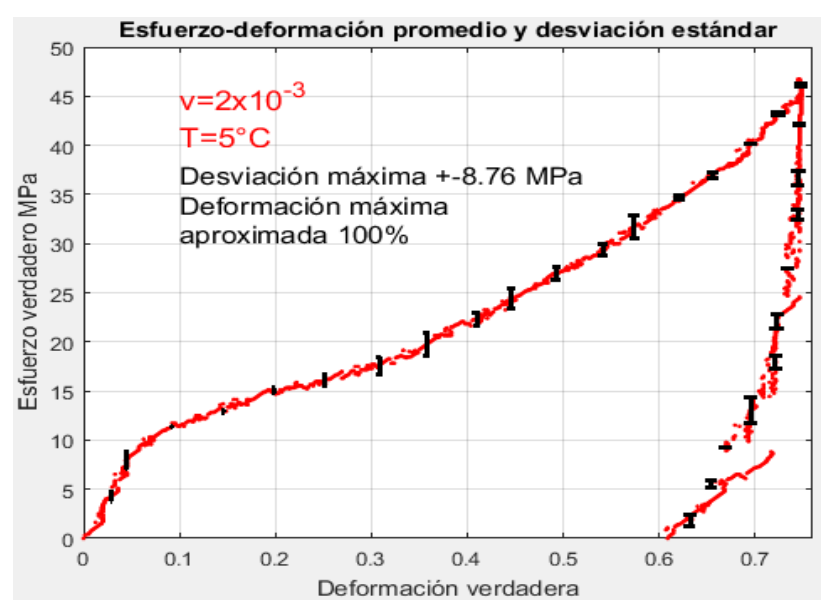

Figura 21 Curva esfuerzo deformación promedio y barras de error expresado por su desviación estándar

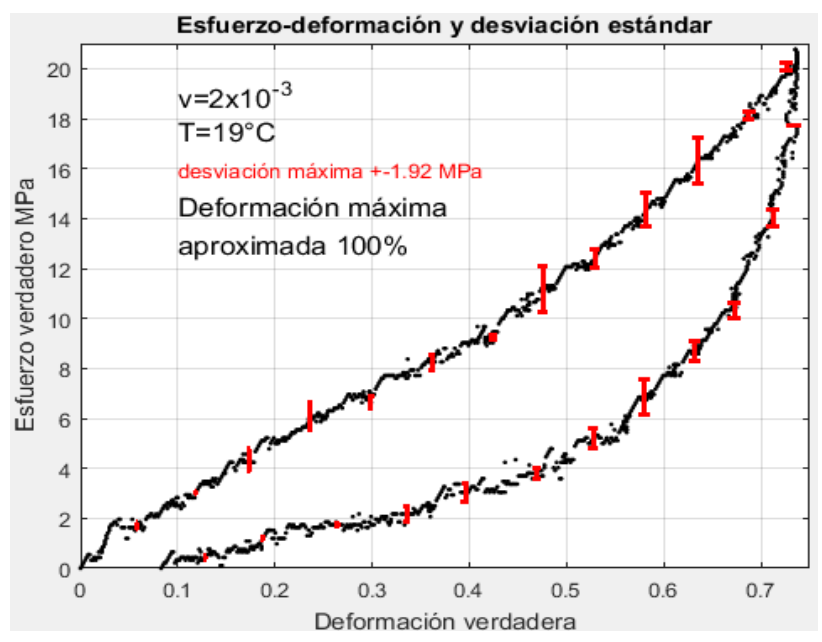

Figura 22 Curva esfuerzo deformación promedio y barras de error expresado por su desviación estándar

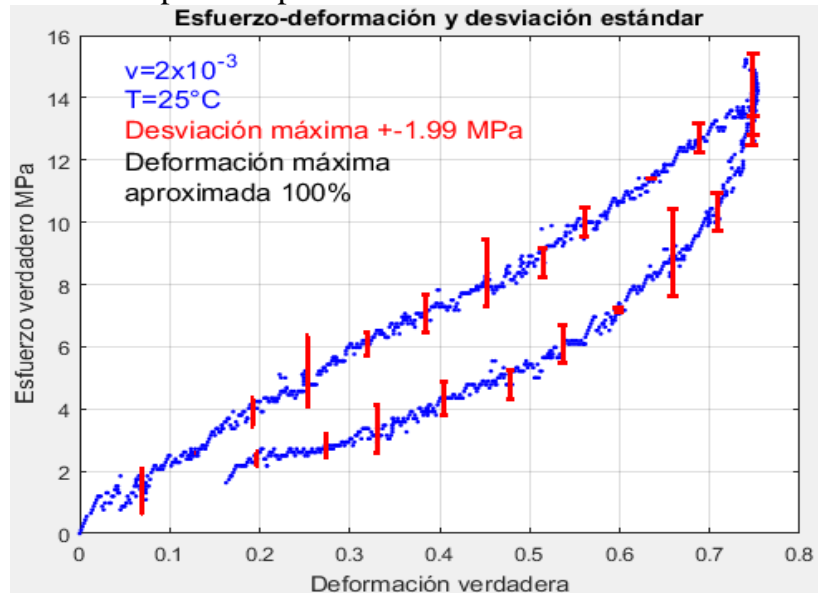

Figura 23 Curva esfuerzo-deformación promedio y barras de error expresado por su desviación estándar 


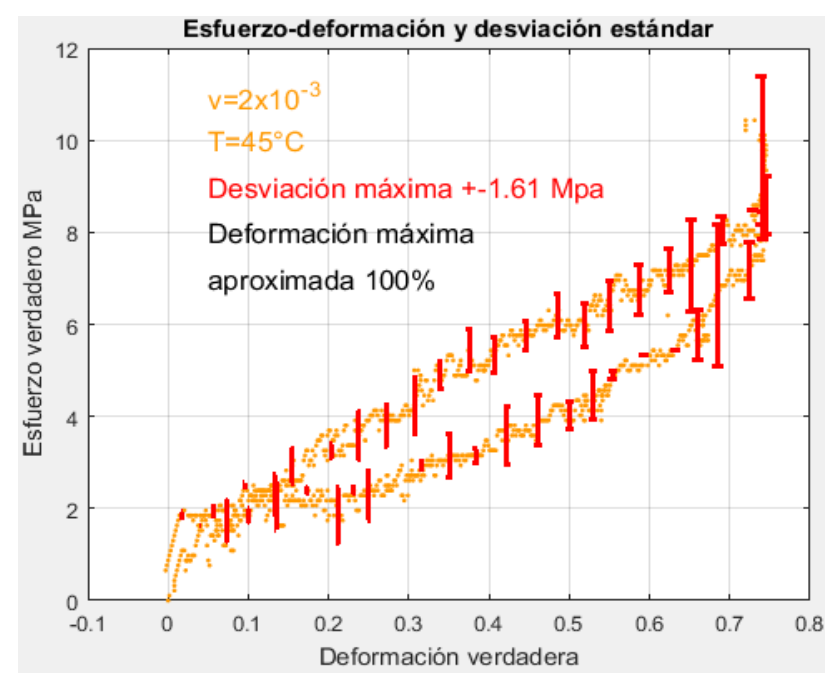

Figura 24 Curva esfuerzo-deformación promedio y barras de error expresado por su desviación estándar

\section{Conclusiones}

Utilizando este procedimiento se obtiene información experimental de 19 curvas esfuerzo-deformación para una barra del polímero PU-SMP MM2520, ello sin contar con los instrumentos de laboratorio. Estos resultados por sí solos no parecen representar una aportación importante sin embargo considerando las posibilidades que ofrece contar con estos vale la redimensionar la importancia de contar con ellos, por ejemplo, se puede desarrollar el modelado del comportamiento del PU-SMP MM2520 para predecir los esfuerzos a diferentes velocidades de deformación y diferentes temperaturas junto con su propiedad de recuperación de forma, con el fin de ser utilizado como material de fabricación para actuadores o sensores en sistemas mecatrónicos o sistemas automáticos.

Con el fin de validar el modelo constitutivo que se proponga es necesario contar con datos para comparar y esos datos deben ser obtenidos de manera experimental, para ello el procedimiento descrito en este trabajo aumentan las opciones para contar con datos experimentales. pues una de ellas es buscar tener contacto con los investigadores que posean los datos de interés o emplear el procedimiento aquí propuesto para extraerlos de las publicaciones, la primer opción es la que ofrece mayor facilidad para conseguir datos experimentales, sin embargo no siempre será posible conseguir directamente con los investigadores este tipo de datos debido principalmente a lo reciente de la investigación y esto por el potencial en cuanto desarrollo tecnológico que puedan ofrecen los datos en cuestión.

Si bien en el presente documento se aplica la técnica de segmentación de imágenes a un grupo específico de gráficos con el fin de obtener los valores de las curvas, el método se puede aplicar a cualquier gráfico digitalizado. Los datos así extraídos pueden ser de utilidad en una etapa intermedia o final en un proceso de investigación como información para validar una hipótesis, modelo constitutivo o diseño.

Es importante recordar que el procedimiento propuesto, para obtener datos, se alimenta de información generada u obtenida por terceros, por lo que no se debe olvidar citar a las fuentes de las que se obtiene las imágenes de donde se extrae la información, así como dar el crédito a los investigadores responsables de las mismas.

\section{Referencias}

[1] E. A. Pieczyska, M. Maj, K. KowalczykGajewska, M. Staszczak, A. Gradys, M. Majewski. M. Cristea. H. Tobushi, H. Hayashi. (2015) Thermome chanical properties of polyurethane series shape memory polymers experiment and modelling. J. Smart Materials and Structures.

[2] R. González y R. E. Woods, Digital Image Processing, Prentice Hall. 2002.

[3] Roberto Rodriguez Morales, Juan Humberto Sossa Azuela. (2012). Procesamiento y Análisis Digital de Imágenes. Alfaomega Ra-Ma. Dr. Isidoro Olvera (Eje 2 Sur) No. 74, Col. Doctores, 06720. Ciudad de México.

O Marques. (2011). PracticalImage and Video Processing Using MATLAB. New Jersey US. WILEY

Peza O., E. (2019). Uso del Computo para la simulación de la deformación y recuperación de forma en una barra de PU-SMP MM2520. Tesis de maestría. Universidad Nacional Autónoma de México, CDMX.

Tony F. Chan, Lummita A. Vese. (2001).Active Contour Without Edges, IEEE Transactions on Image Processing. Vol. 10, No. 2, pp 266-277. 
M. Kass, A. Witkin, and D. Terzopoulos, "Snakes: Active contour models," Int. J. Comput. Vis., vol. 1, pp. 321-331, 1988.

S. Byers and A. Raftery, "Nearest-neighbor cluster removal for estimating features in spatial point processes," J. Amer. Statist. Assoc., vol. 93, no. 442, pp. 577-584, 1998.

N. Paragios and R. Deriche, "Geodesic Active Regions for Texture Segmentation," INRIA RR3440, 1998. Amar Mitiche, Ismayl Ben Ayed. (2010). Varational and Level set methods in Image segmentation, Springer-VerlagBerlin Heidelberg.

Erik Cuevas, Daniel Zaldivar, Marco Pérez. (2015). Procesamiento Digital de Imágenes con MATLAB y Simulink. Alfaomega Ra-Ma. Dr. Isidoro Olvera (Eje 2 Sur) No. 74, Col. Doctores, 06720. Ciudad de México.

MathWorks®. (2018) What Is Image segmentation? 3 things you need to know recuperado de https://www.mathworks.com/discovery/imagesegmentation.html 